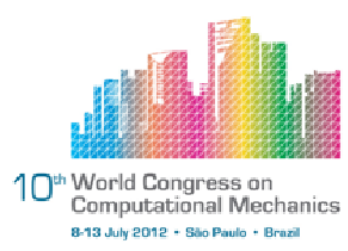

\title{
NUMERICAL STUDY OF TUBE-BUNDLE FLOW-INDUCED VIBRATIONS WITH MULTIPHASE-POD APPROACH
}

\author{
Marie Pomarède ${ }^{1}$, Erwan Liberge ${ }^{2}$, Aziz Hamdouni ${ }^{2}$, Elisabeth Longatte ${ }^{1}$, Jean-François \\ Sigrist $^{3}$ \\ ${ }^{1}$ LaMSID - UMR CNRS \# 8193, 1 Avenue du Général de Gaulle 92141 Clamart, FRANCE \\ ${ }^{2}$ LaSIE - FRE CNRS 3474, Avenue Michel Crépeau 17042 La Rochelle, FRANCE \\ ${ }^{3}$ DCNS Research, Dept. Dynamique des Structures - CESMAN 44620 La Montagne, FRANCE
}

\begin{abstract}
Fluid-Structure Interactions are present in a large number of systems of nuclear power plants and nuclear on-board stoke-holds. Particularly in steam generators, where tube bundles are submitted to cross-flow which can lead to structure vibrations. We know that numerical studies of such a complex mechanism is very costly, that is why we propose the use of reduced-order methods in order to reduce calculation times and to make easier parametric studies for such problems. We use the multiphase-POD approach, which is an adaptation of the classical POD approach to the case of a moving structure in a flow, considering the whole system (fluid and structure) as a multiphase domain. We are interested in the case of large displacements of a structure moving in a fluid, in order to observe the ability of the multiphase$P O D$ technique to give a satisfying solution reconstruction. We obtain very interesting results for the case of a single circular cylinder in cross-flow (lock-in phenomenon). Then we present the application of the method to a case of confined cylinders in large displacements too. Here again, results are encouraging. An on-going work consist in going further testing parametric studies with POD-Galerkin approach and with POD basis interpolation. A future work will consist in applications to fluid-structure interactions.
\end{abstract}

Keywords: Tube bundle systems, Multiphase-POD, Flow-Induced Vibrations.

\section{INTRODUCTION}

Nuclear power plants and nuclear on-board stokeholds are containing very complex installations where flow-induced vibrations are present at various levels. Particularly, tube bundle systems in the steam generator part are prone to fretting-wear or even breaking, mainly due to transverse flow-induced vibration problems [4, 7, 8, 13, 18, 25, 23, 24]. But, as well as experiments, numerical simulations of fluid-structure interactions in a tube bundle system remain very costly $[5,15,28]$ : in order to give a relevant description of the flow and of the structure displacements, it is necessary to construct a system with a large number of degrees of freedom. The three-dimensional aspect of the flow and of the fluid-structure interactions 
added to the large number of tubes and their reciprocal interactions force us to lead long-time calculations, in order to be as precise as possible.

One of the existing alternatives to these restrictive situations from an industrial point of view is to have recourse to reduced-order models $[1,11,2,9,19,21]$ such as POD (Proper Orthogonal Decomposition) $[29,14,17]$. It is thus possible to make numerical calculations in a very short time, and this paves the way to lead parametric studies or even real-time control. Here, the challenge consists in the adaptation of POD to the case of fluid-structure interaction problems. Liberge \& Hamdouni $[19,20]$ proposed an efficient way to cope with the moving fluid-structure interface; this method is called "Multiphase-POD" : the idea is to consider the whole system (fluid and structure domains) as a unique multiphase domain. The advantages of this technique are numerous. First, the data can be extracted from any way (experiments, moving grid techniques, etc.), it is only necessary to know how data are organized to proceed to the interpolation. Here, we show its ability to reproduce large structure displacements, which is often, to the author's knowledge, not so easy with other reduced-order models. The first part of this paper is dedicated to the description of the Multiphase-POD method. Then, its application to the case of large displacements of a single circular cylinder under cross-flow (lock-in phenomenon) is presented in a second part. Finally, a 2D tube-bundle configuration is considered with one moving cylinder under cross-flow.

\section{MULTIPHASE-POD}

We consider here that POD-Galerkin method is well known (see for example [14]) and we are interested on its adaptation to FSI through Multiphase-POD. Complete calculations are leaded with a classic ALE approach [12]. Thus, in this case of Flow Induced Vibrations, classic POD-Galerkin method cannot be used because of the presence of a moving interface : POD modes are only spatial and consequently, they do not contain any dynamic information, although snapshots, in the case of an ALE calculation, have been taken for several positions of mesh nodes.

To get round this problem, Liberge \& Hamdouni [19] proposed an original method that treats the case of a fluid-structure interaction problem with an adaptation of the POD-Galerkin technique, which is called "Multiphase-POD method", where a non-moving mesh is used. The description of the Multiphase-POD method is the following : lets consider a global domain $\Omega$ containing the fluid domain $\Omega_{f}(t)$ and the solid domain $\Omega_{s}(t)$ at each time step $t$, where the solid domain is considered as a particular fluid with its own physical characteristics (density, viscosity). We have $\Omega=\Omega_{f}(t) \cup \Omega_{s}(t) \cup \Gamma_{i}(t)$, where $\Gamma_{i}(t)$ is the interface between fluid and solid domains. A global velocity field $u \in H(\Omega)$ (with $H$ a Hilbert space) is considered :

$$
u(x, t)=u_{f}(x, t) \chi_{\Omega_{f}}(x, t)+u_{s}(x, t) \chi_{\Omega_{s}}(x, t)
$$

where $\chi_{\Omega_{f}}$ and $\chi_{\Omega_{s}}$ are respectively characteristics functions defining if the considered point position is in the fluid or in the solid domain :

$$
\chi_{\Omega_{s}}(x, t)=\left\{\begin{array}{l}
1 \text { if } x \in \overline{\Omega_{s}} \\
0 \text { if } x \notin \overline{\Omega_{s}}
\end{array}\right.
$$


and $\chi_{\Omega_{f}}(x, t)=1-\chi_{\Omega_{s}}(x, t)$. Taking into account this notations, a global weak form of Navier-Stokes equations on $\Omega$ is made possible to formulate :

$$
\int_{\Omega} \rho \frac{\partial u(x, t)}{\partial t} u^{*} d x+\int_{\Omega}(u \cdot \nabla) u \cdot u^{*} d x=\int_{\Omega}(\nabla \cdot \sigma) u^{*} d x
$$

where $u^{*}$ is a test-function defined as $u^{*} \in H(\Omega)$ with the non-deformable solid constraint :

$$
D\left(u^{*}\right)=0 \text { in } \Omega_{s}(t)
$$

Each field or variable is defined on the global domain $\Omega$ as described below:

$$
\left\{\begin{array}{l}
u(x, t)=u_{f}(x, t) \chi_{\Omega_{f}}(x, t)+u_{s}(x, t) \chi_{\Omega_{s}}(x, t) \\
\sigma(x, t)=\sigma_{f}(x, t) \chi_{\Omega_{f}}(x, t)+\sigma_{s}(x, t) \chi_{\Omega_{s}}(x, t) \\
\rho(x, t)=\rho_{f}(x, t) \chi_{\Omega_{f}}(x, t)+\rho_{s}(x, t) \chi_{\Omega_{s}}(x, t) \\
\mu(x, t)=\mu_{f}(x, t) \chi_{\Omega_{f}}(x, t)+\mu_{s}(x, t) \chi_{\Omega_{s}}(x, t)
\end{array}\right.
$$

Lets define both components of the constraints tensor $\sigma$ :

$$
\sigma_{f, i j}(x, t)=-p \delta_{i}^{j}+2 \mu_{f} D_{i j}\left(u_{f}\right)
$$

where $\delta_{i}^{j}$ is the Kroenecker symbol and $D_{i j}$ is the deformation velocity tensor. The definition of the structural compotent $\sigma_{s}(x, t)$ allows taking into account that the solid has its specific viscosity and the non-deformable structural condition. For the viscosity, a penalization term is used: in order to specify that the domain $\Omega_{s}(t)$ is solid, the viscosity is artificially increased. To insure the non-deformable condition, a Lagrange multiplier $\Lambda$ is added. Thus, the structural component of the constraints tensor is:

$$
\sigma_{s, i j}(x, t)=-p \delta_{i}^{j}+\Lambda+2 \mu_{s} D_{i j}\left(u_{s}\right)
$$

Developing the global weak form with these definitions and making the Proper Orthogonal Decomposition on the global velocity flow field leads to the construction of a dynamical system for the whole domain $\Omega$ which is fixed all over the studied time interval. Taking into account the space-time decomposition of the global velocity field as:

$$
u(x, t)=\sum_{i=1}^{N} a_{n}(t) \Phi_{n}(x)
$$

where $\Phi_{n}, n=1, . ., N$ are elements of the POD basis and $a_{n}(t), n=1, . ., N$ are time coefficients, the final dynamical system is the following :

$$
\begin{cases}\sum_{i=1}^{N} \frac{d a_{i}}{d t} A_{i n}=\sum_{i=1}^{N} \sum_{j=1}^{N} B_{i j n} a_{i} a_{j}+\sum_{i=1}^{N} C_{i n} a_{i}+E_{n} \\ D(u)=0 \text { on } \Omega_{s}(t) & \text { (non deformability) } \\ \frac{\partial \chi_{\Omega_{s}}}{\partial t}+u \cdot \nabla \chi_{\Omega_{s}}=0 & \text { (characteristic function transfer) }\end{cases}
$$

for each $n=1, . ., N$ where $N$ is the number of modes in the POD basis. Coefficients $A_{i n}, B_{i j n}, C_{i n}, E_{n}$ are not detailed here, but a very important point to notice is that they are not 
all exclusively spatial coefficients, because some of them contain the physical characteristics $\rho(x, t)$ and $\mu(x, t)$. Thus, they have to be re-calculated at each time step: the time calculation is increased in comparison with a classic POD model without moving structure. But this time calculation is still less than a complete calculation. Another approach consists in making the proper orthogonal decomposition of the characteristic function $\chi_{\Omega_{f}}(x, t)$ also, which allows avoiding the time dependence of all coefficients of the dynamical system. For more precisions, see [20]. Practical implementation of the Multiphase-POD technique is described below.

1. Lead a complete ALE calculation of the fluid-structure interaction problem during a time interval $[0, \mathrm{~T}]$

2. Extract enough snapshots from this complete calculation

3. Create a unique Cartesian fixed mesh containing both fluid and solid domains

4. Interpolate each extracted snapshot onto the fixed reference mesh: new fixed snapshots are created

5. Apply the classic POD approach for the new snapshots constructed on the reference mesh

6. Construct the dynamical system following (9) and resolve it with a classic method (Runge-Kutta for example).

\section{APPLICATION TO LOCK-IN PHENOMENON OF A SINGLE CIRCULAR CYLIN- DER}

In a first time, we propose a simple application of the Multiphase-POD technique, which is the case of a circular cylinder under cross-flow (see Fig.1). The cylinder is submitted to transverse displacements ( $y$-direction) due to the presence of the flowing fluid. The case of small displacements of the structure has already been tested with Multiphase-POD in [19]. Here, we consider the lock-in phenomenon [16, 26, 30], where amplitudes of the structure displacement are of the order of the cylinder radius. The fluid domain is considered as infinite, as boundaries are far enough from the structure. The effects that the flow exerts on the structure are modeled through a restoring force. Reynolds number is $\mathcal{R}_{e}=100$, fluid is water. Cylinder displacement maximal amplitude is $A^{*}=0.58 D$, where $D$ is the cylinder diameter: the frequency lock-in mechanism is reached.

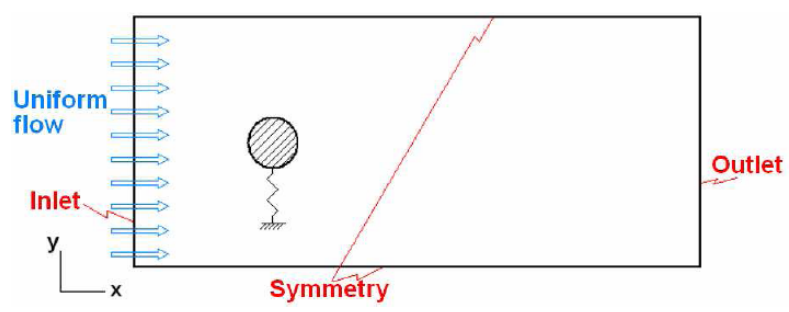

Figure 1. Case of a single circular cylinder and boundary conditions 
Complete calculations are leaded with the CFD code Code_Saturne [3] and data at the interface are interpolated to the cylinder gravity center. The reduced-order model is constructed with the following characteristics: 250 snapshots are extracted from the complete ALE calculation, 6 POD modes are constituting the POD basis. The fixed reference mesh contains $200 \times 250$ points. The dynamical system resolution in the present case is simplified: indeed, the penalization term is sufficient to guarantee the non-deformable condition. Time integration scheme is Runge-Kutta 4.

The two first time coefficients are represented on Fig.2, they are well reconstructed by the reduced model. And, as they are containing the main part of the system energy, this good reproduction allows a good reconstruction of the velocity flow field and the cylinder displacement is also well reproduced (Fig.3), which is confirming that 1) the Multiphase-POD method is able to reproduce a structure displacement and a fluid flow with its global formulation and 2) the Multiphase-POD method is able to reproduce large displacements of the structure. The latter point is interesting for the willingness of studying instability behaviors.
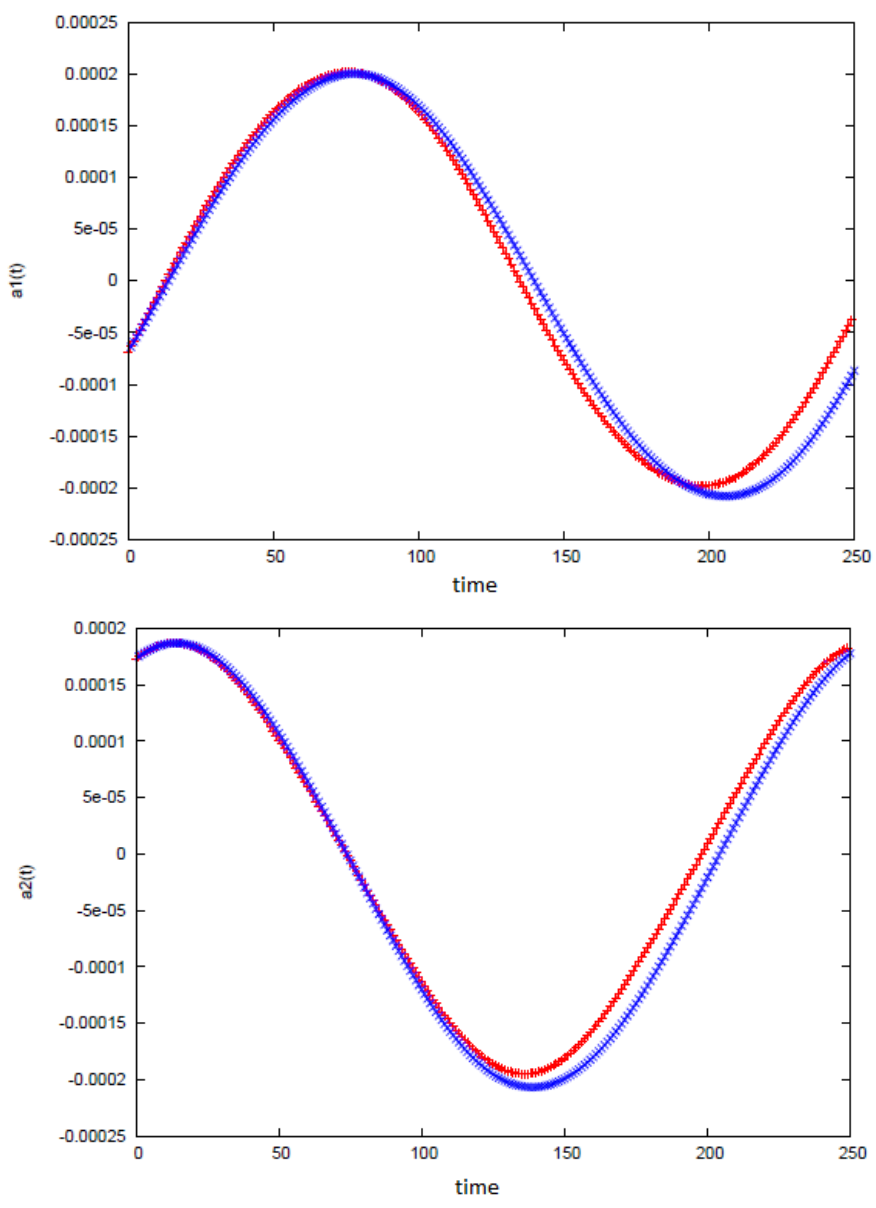

Figure 2. Two first time coefficients of the velocity field for the single cylinder. +++ direct coefficient; xxx Multiphase-POD reconstruction 


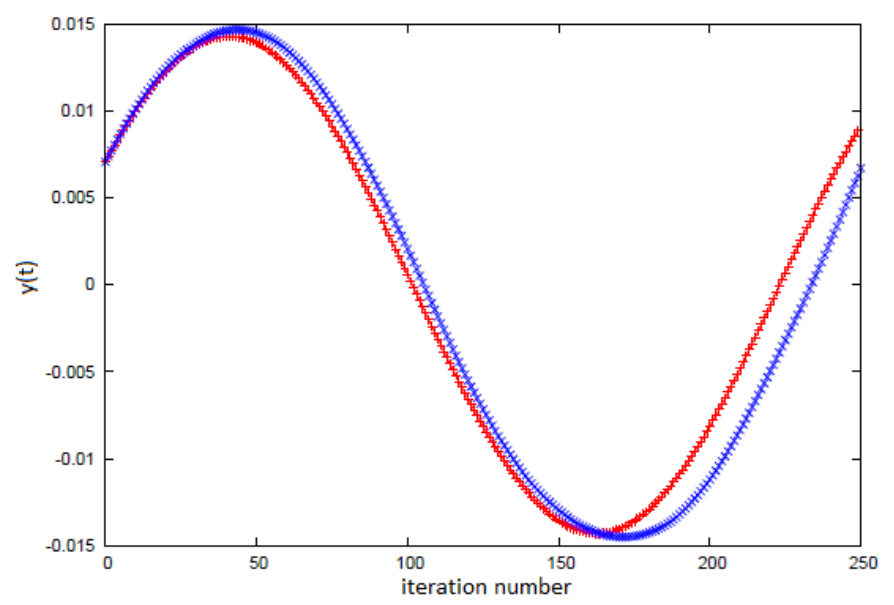

Figure 3. Gravity center displacement reconstruction of the cylinder. +++ direct calculation; xxx Multiphase-POD reconstruction

\section{APPLICATION TO FIV IN TUBE-BUNDLE CONFIGURATION}

In order to consider a configuration close to the case of a tube bundle of heat exchanger, we consider a circular cylinder in a confined configuration. Non-dimensional numbers are adapted to this configuration, here Reynolds number is defined as $\mathbf{R}_{e}=\frac{\rho U_{p} D}{\mu}$. The step fluid velocity $U_{p}$ takes into account the tube confinement and is defined as: $U_{p}=U_{\infty} \frac{P}{P-D}$ where $U_{\infty}$ is the equivalent mean flow velocity that would have been imposed in an infinite domain and $P$ is the pitch ratio (distance between two neighbouring cylinders centres). Geometry and boundary conditions are depicted on Fig.4: a 2D domain and only one tube and its neighbors are considered, with inlet/outlet boundary conditions. Thus, the domain is not representing a whole tube bundle but a confined case. Reynolds number is fixed to $\mathcal{R}_{e}=2000$, complete calculation is also leaded with Code Saturne which has been validated in various FSI studies in tube bundle systems $[5,15,22]$. Large displacements in the $y$-direction (see Fig.4) of the central cylinder are considered $\left(A^{*}=0.35 D\right.$ when $\left.P / D=0.44 D\right)$. The reference fixed mesh contains $200 \times 200$ points. 


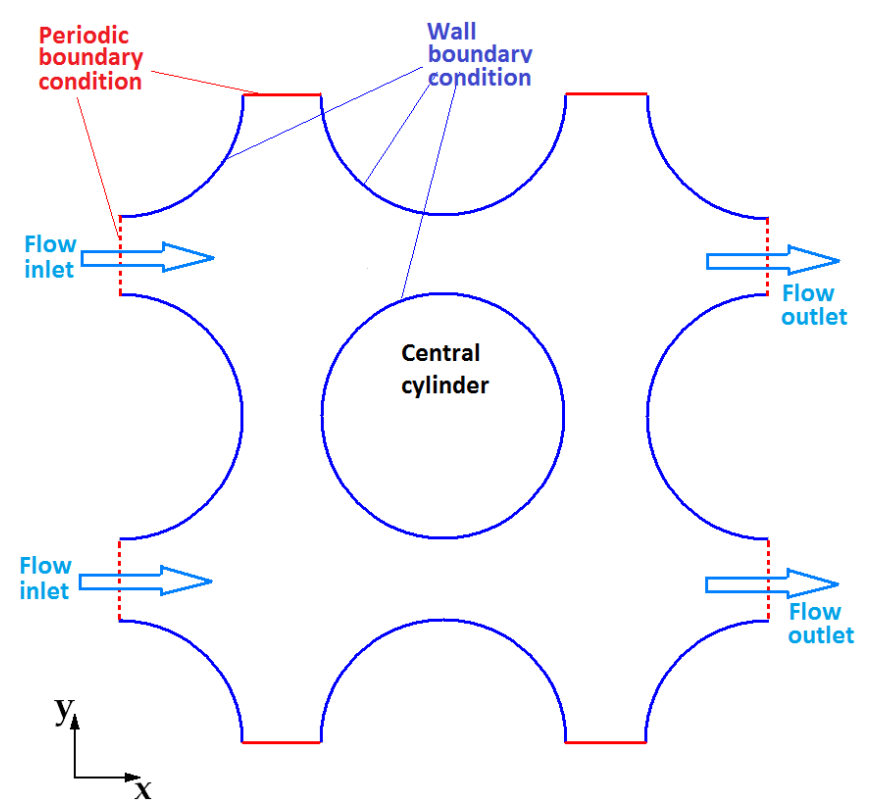

Figure 4. Boundary conditions for the confined tube

Figure 5 represents the comparison between the global velocity flow field from the complete calculation and the interpolated velocity flow field. It allows to check the precision of the snapshots interpolation algorithm: velocity levels are well reproduced after interpolation. In the interpolated case (right-hand side), the non-zero velocity in the cylinder zone is representative of the structure velocity, which is now considered as the second phase of the flow.

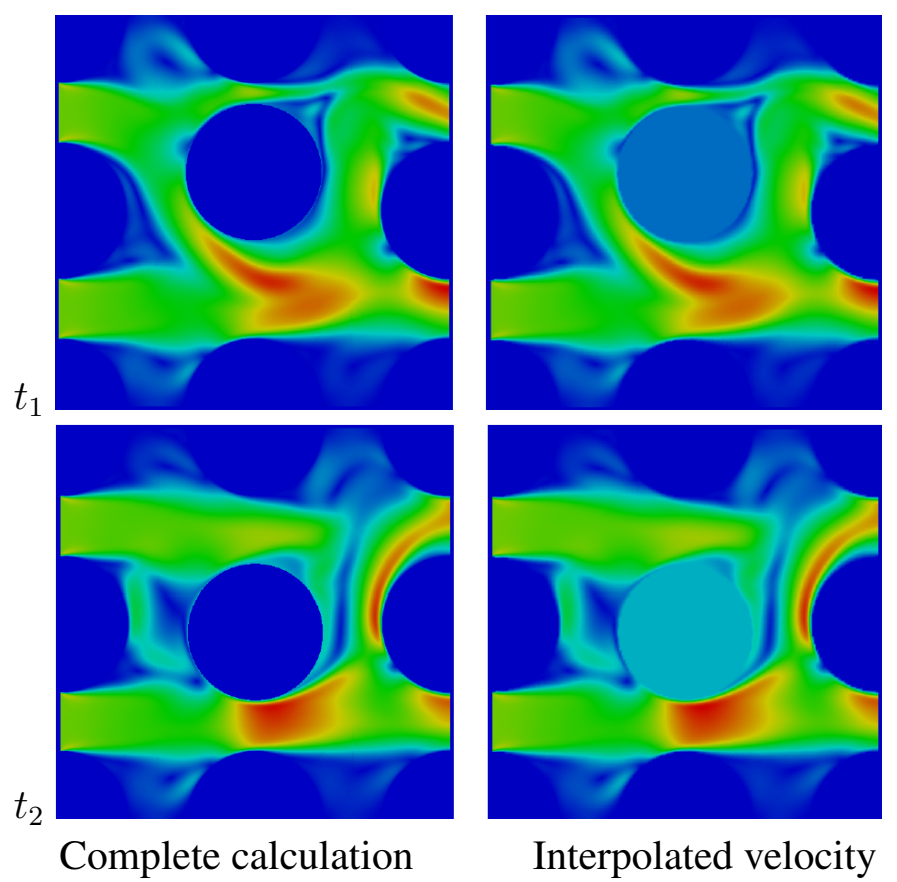

Figure 5. Comparison between complete and interpolated velocity field at two dates $t_{1}$ and $t_{2}$ 
Figure 6 shows the comparison between the central cylinder displacement calculated by complete calculation and by Multiphase-POD. The reconstruction gives very satisfying results, which is confirmed by the observation of the two first time coefficients of the global velocity flow field (Fig.7). The reconstruction of large displacements with Multiphase-POD in the case of a confined tube bundle is very interesting. It allows to plan for its implementation to unstable fluid-structure interactions like fluid-elastic instability occurring in tube bundle systems $[6,10,18,25,27]$.

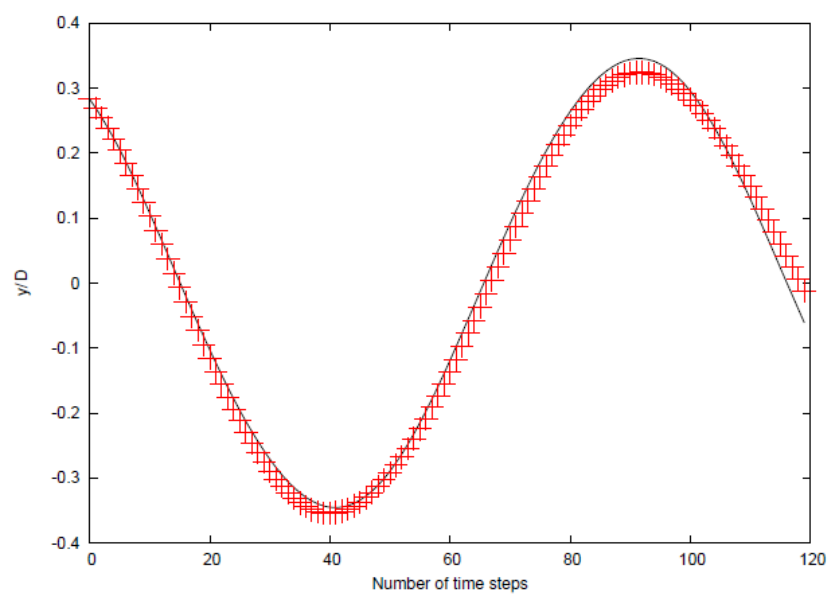

Figure 6. Comparison between complete and multiphase-POD reconstruction of the central cylinder displacement. — complete calculation; +++ Multiphase-POD reconstruction 

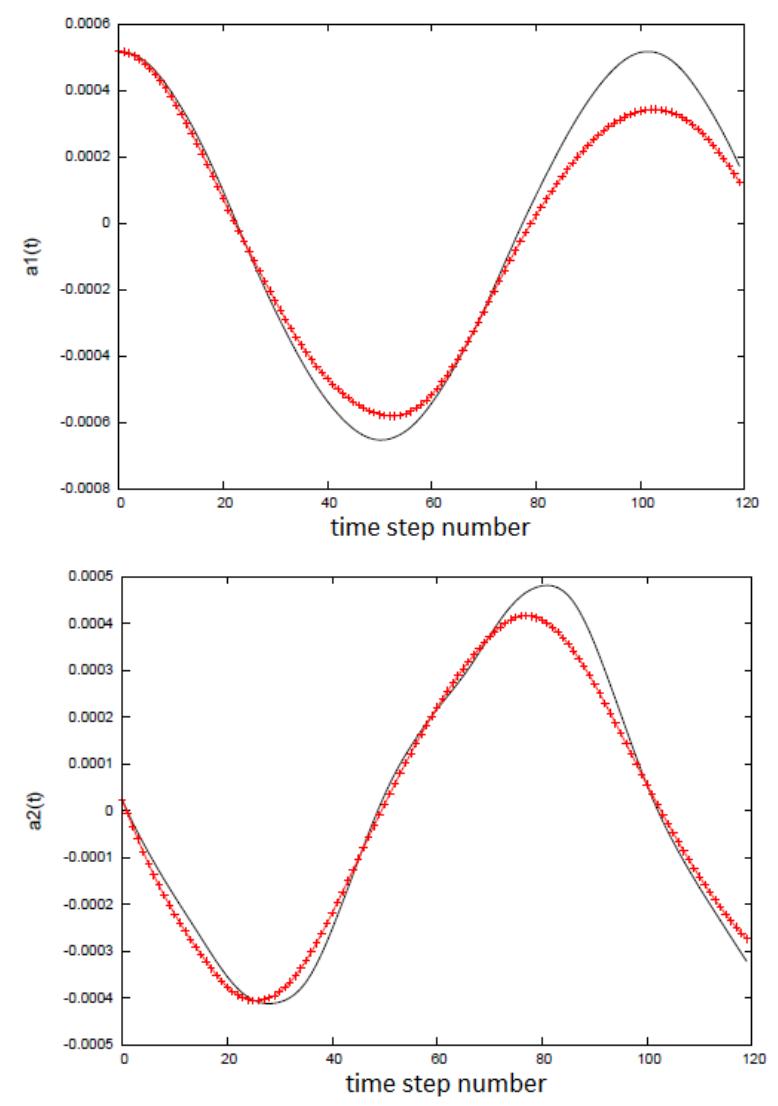

Figure 7. Comparison between complete and multiphase-POD reconstruction of time coefficients. +++ direct coefficient; - Multiphase-POD reconstruction

\section{CONCLUSION}

In this paper, the Multiphase-POD method is presented and applied to the case of a single circular cylinder moving under cross-flow and a confined cylinder in tube bundle under cross-flow. The method was already shown to be efficient in the case of small displacements of a structure under flow sollicitations and here, we show its efficiency in the case of large displacements of the structure. This is a very interesting point in order to treat instabilities that can appear in a large number of industrial systems. Moreover, a simple case of tube bundle has been successfully past with Multiphase-POD, which is encouraging for reducing calculation times in this context. An on-going work is the application of parametric studies with the help of POD: the main interest of reduced-order models consists in their ability to reconstruct various solutions of a system where one or several parameters have been changed. Indeed, the reconstruction of a solution for which we already have the complete calculation is not sufficient. Further work will consist in the application of these methods to the case of FSI. 


\section{References}

[1] Allery, C., Guerin, S., Hamdouni, A., and Sakout, A. Experimental and numerical POD study of the Coanda effect used to reduce self-sustained tones. Mechanics Research Communications, 31:105-120, January-February 2004.

[2] Anttonen, J., King, P., and Beran, P. Application of Multi-POD to a pitching and plunging airfoil. Mathematical and Computer Modelling, 42:245 - 259, 2005.

[3] Archambeau, F., Mechitoua, N., and Sakiz, M. Code_Saturne: a finite volume code for the computation of turbulent incompressible flows Industrial applications. International Journal of Finite Volumes, [www.latp.univ-mrs.fr/IJFV/], 1, 2004.

[4] Axisa, F., Antunes, J., and Villard, B. Random excitation of heat exchanger tubes by cross-flows. Journal of Fluids and Structures, 4:231 - 341, 1990.

[5] Benhamadouche, S. and Laurence, D. LES, coarse LES, and transient RANS comparisons on the ow across a tube bundle. International Journal of Heat and Fluid Flow, $24: 470-479,2003$.

[6] Blevins, R. Fluidelastic whirling of a tube rows. ASME Journal of Pressure Vessel Technology, 96:263 - 267, 1974.

[7] Blevins, R. Flow-Induced Vibrations. Van Nostrand Reinhold, 1990.

[8] Blevins, R. and Bressler, M. Acoustic resonances in heat exchangers, Part II: Prediction and suppression of resonance. ASME Journal of Pressure Vessel Technology, 109:282288, 1987.

[9] Bogaers, A. Reduced Order Modeling Techniques for Mesh Movement Strategies as Applied to Fluid Structure Interactions. Master of engineering, University of Pretoria, 2010.

[10] Chen, S. Instability mechanisms and stability criteria of a group of circular cylinders subject to cross-flow, Part I Part II. Journal of vibration, acoustics, stress and reliability in design, 105:51-58, 1983.

[11] Couplet, M., Basdevant, C., and Sagaut, P. Calibrated reduced-order POD-Galerkin system for fluid flow modelling. Journal of Computational Physics, 207:192-220, 2005.

[12] Donea, J., Huerta, A., Ponthot, J., and Rodriguez-Ferran, A. Arbitrary LagrangianEulerian Methods, Encyclopedia of Computational Mechanics Vol. 1: Fundamentals, Chap. 14. Wiley \& Sons, 2004.

[13] Feenstra, P., Weaver, D., and Nakamura, T. Vortex shedding and fuidelastic instability in a normal square tube array excited by two-phase cross-flow. Journal of Fluids and Structures, 17:793-811, 2003. 
[14] Holmes, P., Lumey, J., and Berkooz, G. Turbulence, Coherent Structures, Dynamical Systems and Symmetry. Cambridge Monographs on Mechanics, Cambridge University Press, 1996.

[15] Huvelin, F. Couplage de codes en interaction fluide-structure et application aux instabilités fluide-élastiques. PhD Thesis, Ecole des Sciences Pour l'Ingénieur de Lille, 2008.

[16] Khalak, A. and Williamson, C. Motions, Forces and Mode Transitions in Vortex-Induced Vibrations at Low Mass Damping. Journal of Fluids and Structures, 13:813-851, 1999.

[17] Kunisch, K. and Volkwein, S. Galerkin Proper Orthogonal Decomposition methods for parabolic systems. Numerische Mathematik, 90:117-148, 2001.

[18] Lever, J. and Weaver, D. On the stability of Heat Exchanger Tube Bundles, Part II: Numerical results and comparison with experiments. Journal of Sound and vibration, 107(3):393-410, 1986.

[19] Liberge, E. and Hamdouni, A. Reduced-order modelling method via Proper Orthogonal Decomposition (POD) for flow around an oscillating cylinder. Journal of Fluids and Structures, 26(2):292-311, 2010.

[20] Liberge, E., Pomarède, M., and Hamdouni, A. Reduced-order modelling method by POD-multiphase approach for fluid-structure interactions. European Journal of Computational Mechanics, 19:41-52, 2009.

[21] Lieu, T., Farhat, C., and Lesoinne, M. Reduced-order fluid/structure modelling of a complete aircraft configuration. Computer Methods in Applied Mechanics and Engineering, 195:5730-5742, 2006.

[22] Longatte, E., Bendjeddou, Z., and Souli, M. Methods for numerical study of tube bundle vibrations in cross-flows. Journal of Fluids and Structures, 18:513-528, 2003.

[23] M.P. Païdoussis. A review of flow-induced vibrations in reactors and reactor components. Nuclear Engineering and Design, 74:31-60, 1982.

[24] Pettigrew, M. and Gorman, D. Vibration of heat exchanger tube bundles in liquid and two-phase cross-flow. Flow-induced vibration design guidelines P.Y. Chen edition, ASME PVP, 52:89-110, 1981.

[25] Pettigrew, M. and Taylor, C. Fluidelastic Instability of heat exchanger tube bundle: review and design recommendations. Journal of Pressure Vessel Technology, 113:242_ 256, 1991.

[26] Pomarède, M., Longatte, E., and Sigrist, J.-F. Benchmark of numerical codes for coupled csd/cfd computations on an elementary vortex induced vibration problem. Pressure Vessel and Piping,, July 2009. Prague. 
[27] Price, S. A review of theoretical models for fluidelastic instability of cylinder arrays in cross-flow. Journal of Fluids and Structures, 9:3147-3170, 2001.

[28] Sigrist, J. and Abouri, D. Numerical simulation of a non-linear coupled fluid-structure problem with implicit and explicit coupling procedures. Pressure Vessel and Piping, July 2006. Vancouver.

[29] Sirovich, L. Turbulence and the dynamics of coherent structures, Parts I-III. Quarterly of Applied Mathematics, 45(3):561-590, 1987.

[30] Williamson, C. and Roshko, A. Vortex formation in the wake of an oscillating cylinder. Journal of Fluids and Structures, 2:355-381, 1988. 